\title{
Focal dystonia in musicians: From phenomenology to therapy
}

\author{
Hans-Christian Jabusch and Eckart Altenmüller
}

University of Music and Drama, Institute of Music Physiology and Musicians' Medicine, Hanover, Germany

\section{Keywords}

musicians' cramp; focal dystonia, treatment, botulinum-toxin, trihexyphenidyl,

\section{ABSTRACT}

Background: Musician's dystonia is a task-specific movement disorder which manifests itself as a loss of voluntary motor control in extensively trained movements. In many cases, the disorder terminates the careers of affected musicians. Approximately $1 \%$ of all professional musicians are affected.

Etiology and Pathophysiology: The pathophysiology of the disorder is still unclear. Findings include (a) reduced inhibition in different levels of the central nervous system, (b) maladaptive plasticity, e.g. in the somatosensory cortex and in the basal ganglia, and (c) alterations in sensorimotor processing. Epidemiological data demonstrated a higher risk for those musicians who play instruments requiring maximal fine-motor skills. For instruments where workload differs across hands, focal dystonia appears more often in the more intensely used hand. In psychological studies, musicians with dystonia had more perfectionist tendencies than healthy musicians. These findings strengthen the assumption that behavioral factors may be involved in the etiology of musician's dystonia. Hereditary factors may play a greater role than previously assumed. Preliminary findings suggest a genetic contribution to focal task-specific dystonia with phenotypic variations including musician's dystonia.

Treatment: Treatment options for musician's dystonia include pharmacological interventions such as administration of Trihexyphenidyl or Botulinum Toxin-A as well as retraining programs and ergonomic changes in the instrument. A long-term follow-up study was performed in 144 patients with musician's dystonia. The outcome was revealed on average 8.4 years after onset of symptoms. Outcome was assessed by patients' subjective rating of cumulative treatment response and response to individual therapies. Seventy-seven patients (54\%) reported an alleviation of symptoms: $33 \%$ of the patients with Trihexyphenidyl, $49 \%$ with Botulinum Toxin, $50 \%$ with pedagogical retraining, $56 \%$ with unmonitored technical exercises, and $63 \%$ with ergonomic changes. In embouchure dystonia, only $15 \%$ of patients reported improvement.

The results demonstrate that the situation of musicians with focal hand dystonia may be significantly improved. Positive results after retraining and unmonitored technical exercises underline the benefit of an active involvement of patients in the treatment process. Only exceptionally, however, can musicians with focal dystonia return to normal motor control using the currently available therapies.

\section{INTRODUCTION}

Focal dystonia in musicians, also known as musician's cramp, is a task-specific movement disorder which presents itself as a painless muscular incoordina-

Correspondence concerning this article should be addressed to University of Music and Drama, Institute of Music Physiology and Musicians' Medicine Hohenzollernstrasse 47, 30161 Hannover, Germany, phone: +49 511 3100552, fax: +49 511 3100557, e-mail: jabusch@hmt-hannover.de, altenmueller@hmt-hannover.de 
tion or loss of voluntary motor control of extensively trained movements while a musician is playing the instrument (Jankovic \& Shale, 1989; Lederman, 1991; Brandfonbrener, 1995; Frucht et al., 2001; Altenmüller, 2003). For those who are affected, focal dystonia is highly disabling and in many cases terminates musical careers. According to estimates, one percent of all musicians are affected (Altenmüller, 2003). In this article, the phenomenology and epidemiology of musician's dystonia shall be addressed on the basis of data from 144 affected musicians diagnosed and followed-up at the Institute of Music Physiology and Musicians' Medicine of the Hannover University of Music and Drama. These data will be compared to data from other reports, and discussed with regard to risk factors for the development of musician's dystonia. Treatment strategies and results will be outlined based on a retrospective inquiry and self-evaluation of affected musicians treated with currently available therapeutic approaches.

All 144 patients were professional musicians diagnosed with focal dystonia at our outpatient clinic between 1994 and 2001. The diagnostic procedure during their first visit included a complete neurological examination as well as visual inspection while patients were playing their instruments. None of the patients were suffering from secondary dystonias or other neurological disorders. Data were statistically analyzed using $\chi^{2}$-tests and Fisher's exact tests to identify disproportionate frequencies in the clinical manifestation of subgroups of patients. The level of significance was set at $p=.05$.

\section{DEMOGRAPHICS, EPIDEMIOLOGY AND PHENOMENOLOGY}

The patient group consisted of 116 male (81\%) and 28 female musicians (19\%). The mean age at onset of symptoms was 33 years (range 17-63 years), the mean duration of dystonic symptoms at the time of the first visit was 5.1 years (range $0.1-28$ years). At the time of the onset of symptoms, 74 patients $(51 \%)$ had professional positions as soloists and $24(17 \%)$ were tutti players in orchestras, 25 patients $(17 \%)$ had teaching positions and 21 (15\%) were students. Concerning the genre, 137 patients (95\%) were classical musicians, 2 patients ( $1 \%$ ) were jazz musicians, and 5 patients (4\%) were pop musicians. Nine patients $(6 \%)$ had a history of either writer's cramp or musician's dystonia in their family. The distribution of instrument groups was as follows: 22 patients (15\%) were bowed string players, 40 patients (28\%) were keyboard instrumen- talists, 29 patients ( $20 \%$ ) were playing plucked instruments, 37 patients (26\%) were woodwind players and 16 patients $(11 \%)$ were brass players. The distribution of individual instruments is displayed in Figure 1. In order to compare the distribution of instruments among dystonic musicians with that in a normal population of instrumentalists, the respective numbers of members in the German Orchestra Union were used according to the March-2004 statistics of the German Orchestra Union (representing over $90 \%$ of German orchestra musicians). In our patient group, 20 high string players (violin, viola) and 2 low string players (violoncello, double-bass) were affected. This ratio was compared with the distribution of 3810 high and 1464 low string players in the German Orchestra Union. Taking into consideration that one low and 10 high string players from our patient group were members of the German Orchestra Union, the comparison of 3800 high and 1463 low string players from the Orchestra Union with the distribution in our patient group revealed an overrepresentation of high string players among patients with musician's dystonia $\left(\chi^{2}=3.83, d f=1, p=.05\right)$. 124 patients were suffering from focal upper limb dystonias ( $86 \%)$, which presented themselves mostly as hand dystonias in the typical manner with involuntary cramping of one or more fingers while patients were playing; one trombone player displayed dystonic movement patterns in his left upper arm. Embouchure dystonia was present in 20 patients (14\%). Typical patterns of dystonic posture are displayed in Figure 2. Details of the localization of focal dystonia in different instrument families are given in Figure $3 \cdot \chi^{2}$-tests in 115 patients with unilateral hand dystonia revealed a different laterality of focal hand dystonia $\left(\chi^{2}=23.5\right.$, $d f=3, p<.0001)$ : keyboard musicians and those with plucked instruments were primarily affected in the right hand and string players in the left hand (Bonferroni-corrected follow-up $\chi^{2}$-tests; keyboard vs. string players: $\chi^{2}=13.3, d f=1, p<0,01$; keyboard musicians vs. musicians with plucked instruments: $\left.\chi^{2}=13.6, d f=1, p<0,01\right)$. No such lateralization was seen in woodwind players. Bilateral hand dystonia was seen only in keyboard instrumentalists $(n=4)$ and in woodwind players $(n=4)$. Handedness was categorized as right-handed / left-handed / ambidextrous according to preferences in the use of hands in the activities handwriting, eating with spoon, and cutting with scissors. Among the patients with unilateral hand dystonia, there were 108 right-handed patients and 5 left-handed patients, 2 patients were ambidextrous. Seventy-five right-handed patients had right hand dystonia, and 33 right-handed patients had left 
No. of patients

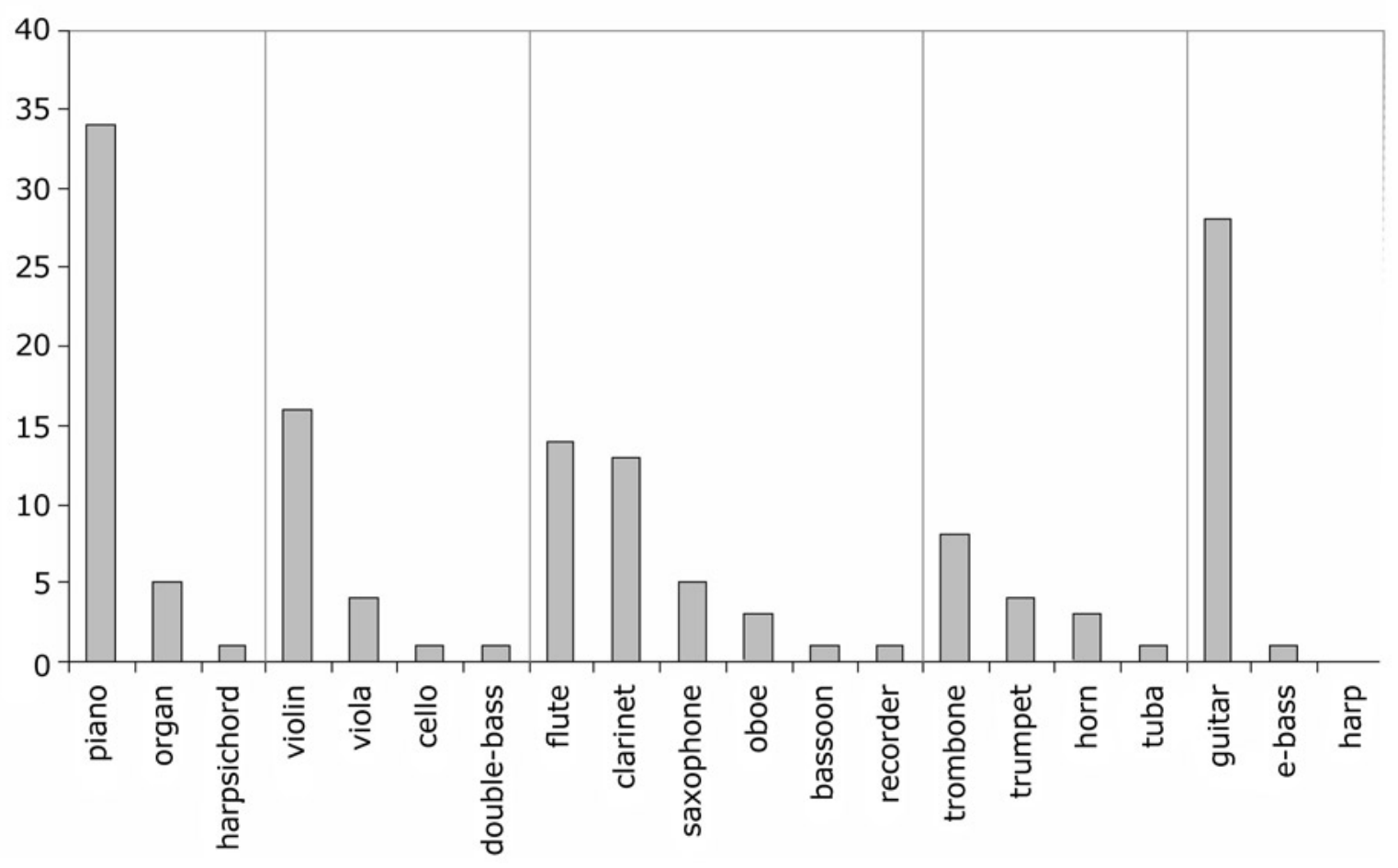

Figure 1.

The distribution of instruments in 144 patients with musician's dystonia. Instrument families are separated by vertical lines.
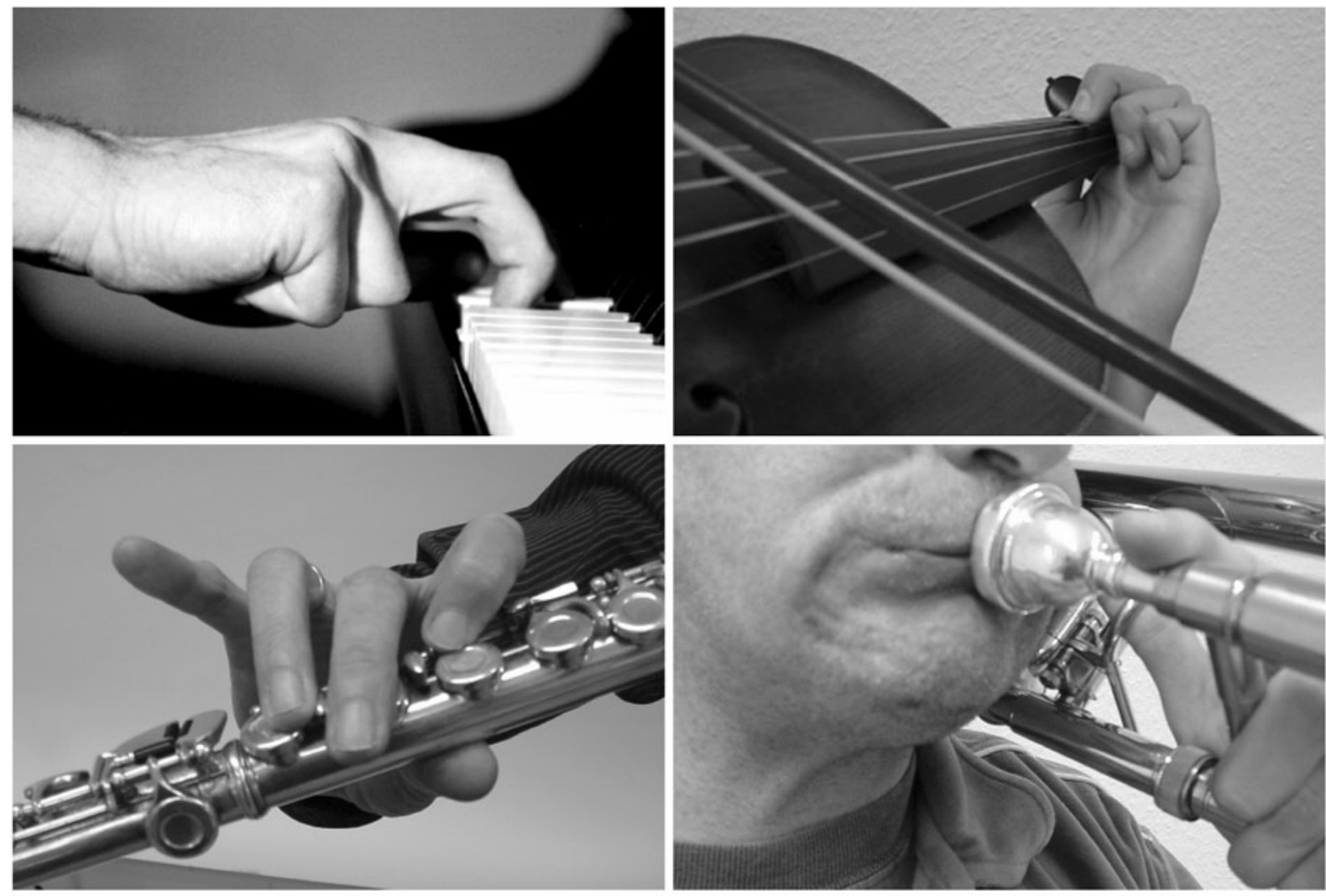

Figure 2.

Typical patterns of dystonic posture in a pianist, a violinist, a flutist and a trombone player. 
No. of patients

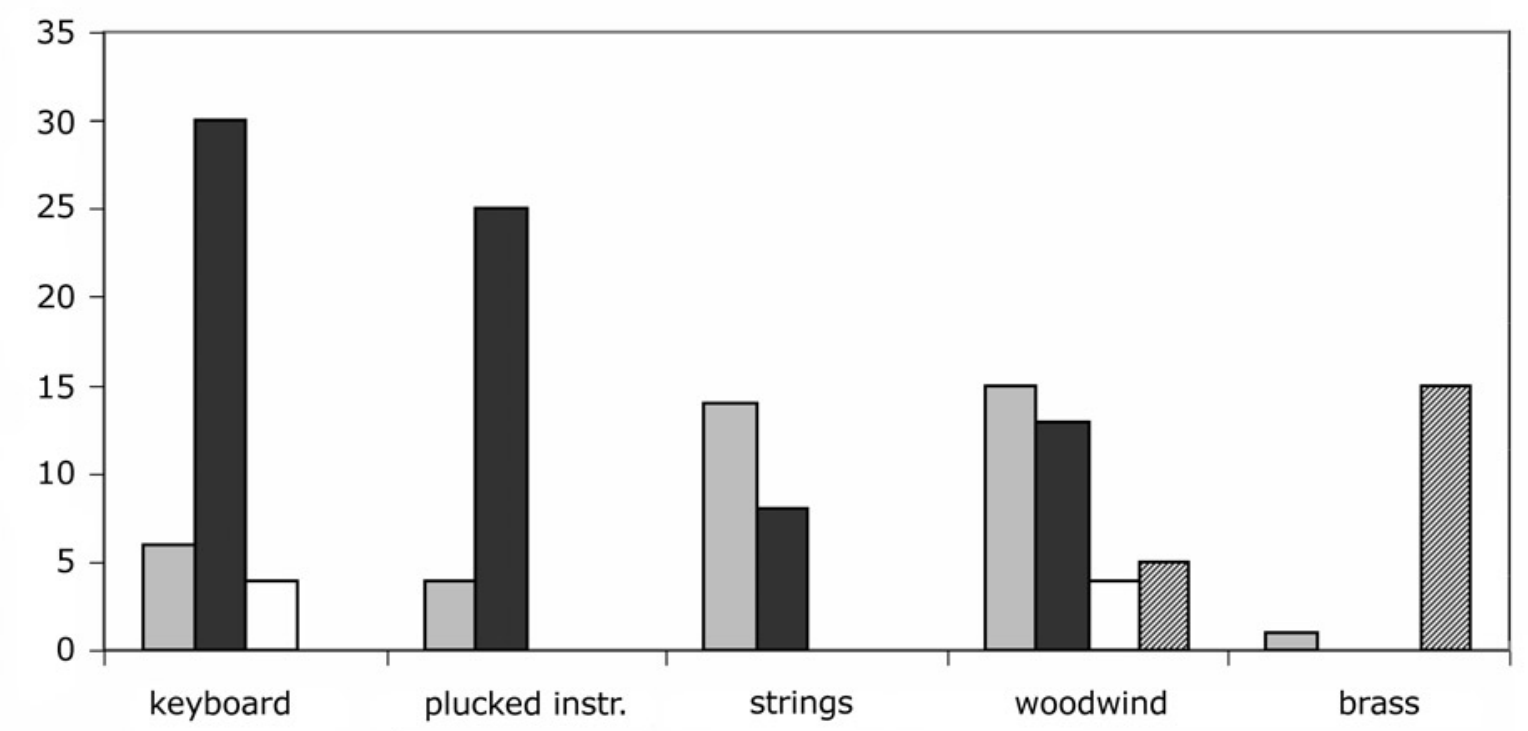

Figure 3.

Localization of musician's dystonia as distributed in different instrument families.

Grey bars: left hand affected. Black bars: right hand affected. White bars: both hands affected. Hatched bars: embouchure dystonia

hand dystonia. This pattern was inverse in left-handed patients: in four of these the left hand was affected (three woodwind players, one guitarist), and in one pianist the right hand was affected. These proportions were significantly different (Fisher's exact test; $p<.05)$.

Ninety-five patients (66\%) had dystonic symptoms only while they were playing the instrument, whereas 49 patients $(34 \%)$ had additional difficulties in other activities such as writing on the computer keyboard or in everyday activities. Local pain preceded focal dystonia in $13(9 \%)$ patients: 7 patients (5\%) experienced local pain only before onset of dystonic symptoms, while $6(4 \%)$ patients had pain in the affected region before and after onset of dystonic symptoms. Twentyfive $(17 \%)$ patients reported local pain only after onset of dystonic symptoms, whereas 106 (74\%) patients never experienced pain in the affected region.

\section{Risk factors for the development of musician's dystonia}

The demographic data demonstrated a preponderance of male musicians in the sample of the present study with a male:female ratio of $4: 1$. In other reports, the male:female ratio of patients with musician's dystonia was between 2:1 (Lederman, 1991) and 5:1 (Lim \& Altenmüller, 2003; the patient group of the latter report partially overlapped with the present sample).
Since female musicians are predominant in the musicians' population in Germany, the latter ratio was corrected to $6: 1$ (Lim \& Altenmüller, 2003). Similarly, this effect has to be taken into account for the noncorrected male:female ratio of $4: 1$ of the present sample of patients, which additionally underlines the increased risk of male musicians to develop focal dystonia. A positive family history of dystonia as seen in $6 \%$ of our patient group, has already been described as a risk factor for development of musician's dystonia in other reports (Jankovic \& Shale, 1989; Altenmüller, 2003; Lim \& Altenmüller, 2003; Schmidt et al, 2006). The mean age at onset of dystonic symptoms (33 years) observed in the present study harmonizes with other studies demonstrating a peak in the fourth decade of life (Lederman, 1991; Brandfonbrener, 1995; Brandfonbrener \& Robson 2002; Altenmüller 2003).

Our results demonstrated an association between the instrument group and the localization of focal dystonia. This is obvious for brass players who are predominantly suffering from embouchure dystonia. Also in musicians with unilateral hand dystonia an association was observed between the instrument group and the localization of focal dystonia. Keyboard musicians (piano, organ, harpsichord) and those with plucked instruments (guitar, e-bass, no harp) were primarily affected in the right hand. All these instruments are characterized by a higher workload in the right hand. Additionally, guitar playing requires higher tempo- 
rospatial precision in the right hand compared to the left hand. Bowed string players who have a higher workload and complexity of movements in the left hand were predominantly affected in the left hand. These observations harmonize with other reports (Tubiana \& Chamagne, 2000; Brandfonbrener \& Robson, 2002; Schuele \& Lederman, 2004). Moreover, focal hand dystonia in both hands was only seen in those musicians with instruments requiring similar movement patterns in both hands (although with different workload), such as woodwind and keyboard instruments. The reported data suggest that musicians with instruments requiring high levels of spatial sensorimotor precision were more often affected by dystonia than were other musicians. Within the string family, musicians who were playing high string instruments (violin, viola) were more often affected compared to those with low string instruments (violoncello, double-bass). Although the comparison of the distribution of string players in our patients group with that of the German Orchestra Union might be biased by unreported cases of musician's dystonia in the union, results suggest that high string players were overrepresented in our patients group. High string instruments require a higher spatial sensorimotor precision in the left hand compared to low string instruments due to the shorter length of their strings: The strings of a violin, for example, are approximately three times shorter than the strings of a double-bass (Fletcher \& Rossing 1991). This results in a threefold decreased distance between the spots of the two tones of a given interval on the fingerboard of the violin and in a higher spatial sensorimotor precision required for the left hand in violinists compared to double-bass players.

Furthermore, in our sample of musicians with unilateral hand dystonia, an association was observed between the handedness and the side affected by dystonia. The majority of right-handed patients were affected in the right hand and the majority of left-handed patients were affected in the left hand. Although the number of left-handed patients was small, this difference was significant and indicated that the hand with a higher total workload tended to be affected. It is noteworthy that there was no string player in the group of left-handed patients. The musical genre of the overwhelming majority of patients was classical music. In contrary to pop or jazz music with improvised structures and great freedom of interpretation, musical constraints are most severe in classical music. The latter requires maximal temporal accuracy in the range of milliseconds which is scrutinized by the performing musician as well as by the audience at any moment of the performance. This, as a consequence, combines the situation of public performance in classical music with a high level of social pressure: the gap between success and failure is minimal in this genre.

Taken together, epidemiological findings indicate that the amount of workload of the respective body part, the complexity of movements and the degree of spatial and temporal sensorimotor precision as well as the level of social constraints associated with the musical performance were related to musician's dystonia.

\section{Pathophysiological findings}

The pathophysiology of focal dystonia is still unclear. Beside of alterations in the basal ganglia circuitry (Naumann et al., 1998; Preibisch et al., 2001) and dysfunctional plasticity in the sensory thalamus (Lenz \& Byl 1999; Hua et al., 2000), there is growing evidence for the abnormal cortical processing of sensory information as well as degraded representation of motor function in patients with focal dystonia (Deuschl et al., 1995; Hallett, 1998; Elbert et al., 1998; BaraJimenez et al., 1998; Pujol et al., 2000). Cortical inhibition was found to be decreased in dystonic patients (Deuschl et al., 1995; Toro et al., 2000; Hummel et al., 2002). In animal studies, repetitive movements induced symptoms of focal hand dystonia and a distortion of the cortical somatosensory representation in monkeys (Byl et al., 1996) suggesting that practice-induced alterations in cortical processing may play a role in focal hand dystonia. Recently, behavioral treatment in patients with focal hand dystonia resulted in clinical amelioration and in reversed somatosensory map distortions giving additional evidence for a practiceinduced cortical map alteration (Candia et al., 2003). The importance of practice and the use of the limb in musician's dystonia was additionally underscored by the finding of improved symptoms after the immobilization of the affected limb in dystonic musicians (Priori et al., 2001). An impressive finding was reported by Rosenkranz et al. (2005), who used focal vibratory input to individual hand muscles to produce sensory input whilst the excitability of corticospinal outputs to the vibrated hand and the other hand muscles was evaluated with transcranial magnetic stimulation. In musicians with dystonia, focal vibratory input to hand muscles resulted in abnormally suppressed short-latency intracortical inhibition of all other hand muscles examined, independent of their functional connectivity with the vibrated muscles. In healthy non-musicians, such vibratory input increased short-latency intracortical inhibition of neighbouring muscles. In 
healthy musicians, however, vibratory input resulted in a suppressed short-latency intracortical inhibition only of those neighbouring muscles that functionally connected with the vibrated muscles. In patients with writer's cramp, short-latency intracortical inhibition of neighbouring muscles was unchanged. It was concluded that the pathophysiology of musician's dystonia differs from that of writer's cramp. Furthermore, it was hypothesized that long hours of practicing complex movements first produce the modulation of sensorimotor interaction seen in healthy musicians, and that this later progresses into the non-focal pattern of shortlatency intracortical inhibition in musician's dystonia (Rosenkranz et al., 2005).

The findings of an increased workload and complexity of movements as well as increased spatial and temporal sensorimotor requirements being related to musician's dystonia harmonize with the hypothesis of a pathomechanism which includes practice-induced alterations. A higher workload in affected musicians might additionally be reflected by the observation of a more perfectionist attitude in dystonic musicians - even before onset of dystonia - compared to other musicians (Jabusch et al., 2004a) and by the finding of a preponderance of soloists $(51 \%)$ in our sample of dystonic musicians. This high rate is in contrast to a rate between $1 \%$ and $8 \%$ of musicians in Germany working as soloists depending on the instrument group (Gembris \& Langner 2005). A perfectionist attitude as well as an outstanding professional position might point at a different and more intense working behavior resulting in a higher workload in those musicians who develop focal dystonia.

Local pain and intensified sensory input due to various causes such as nerve entrapment or trauma or overuse have been described as potential triggers of dystonia (Jankovic \& Shale, 1989; Lederman, 1991; Charness et al.; 1996; Altenmüller, 2003). The parallels of abnormal cortical processing of sensory information and cortical reorganization in patients with focal dystonia and those with chronic pain have already been reported (Flor et al., 1997; Tinazzi et al., 2000; Jabusch et al., 2004a). Local pain preceded focal dystonia in $9 \%$ of the patients in the present study. In this context, it should be emphasized that local pain is not a typical symptom of focal dystonia. Seventy-four percent of the patients of our sample never experienced pain in the affected region. However, as discussed above, pain syndromes may serve as triggers for the development of dystonia and pain may also occur as a consequence of the persistent attempts by patients to correct the involuntary movements and postures.
This may result in painful overuse syndromes in a subgroup of patients.

\section{Possible coaction between predisposition and extrinsic and intrinsic factors}

In view of neurophysiological, epidemiological and psychological findings in musicians with focal dystonia, predisposing factors have been identified such as male gender (Lederman, 1991; Altenmüller 2003; Lim \& Altenmüller 2003) as well as a positive family history (Jankovic \& Shale, 1989; Altenmüller, 2003; Lim \& Altenmüller, 2003; Schmidt et al., 2006), which might constitute a particular vulnerability or susceptibility to musician's dystonia. Additional extrinsic and intrinsic factors may trigger the manifestation of musician's dystonia on the basis of a given susceptibility. Intrinsic triggering factors are physical disorders resulting in local pain and / or intensified somatosensory input (trauma, nerve entrapment, overuse injury) (Lederman, 1991; Charness et al., 1996; Altenmüller, 2003) as well as psychological conditions such as perfectionism and anxiety, which were found to be related to musician's dystonia (Jabusch et al., 2004a). Anxiety has been hypothezised to be an aggravating factor during onset of musician's dystonia, triggering an emotion-dependent mechanism of motor memory consolidation of dystonic movements (Jabusch \& Altenmüller, 2004a). According to Rosenkranz et al. (2005), intracortical disinhibition in musician's dystonia might be an acquired, practice-induced phenomenon. Extrinsic triggering factors, according to epidemiological findings (Tubiana \& Chamagne, 2000; Brandfonbrener \& Robson, 2002; Schuele \& Lederman, 2004), are spatial and temporal sensorimotor constraints as well as musical and social constraints typical of the performance situation in classical music. The possible coaction between predisposition and intrinsic and extrinsic triggering factors in the manifestation of musician's dystonia is displayed in Figure 4.

\section{TREATMENT STRATEGIES AND RESULTS}

For a survey of treatment results, 160 consecutive professional musicians and music students with musician's dystonia were recruited (Jabusch et al., 2005). Treatment strategies included: medication with Trihexyphenidyl; injection therapy with Botulinum Toxin; ergonomic changes; pedagogical retraining; non-specific exercises on the instrument. Outcome data were assessed using a standardized question- 


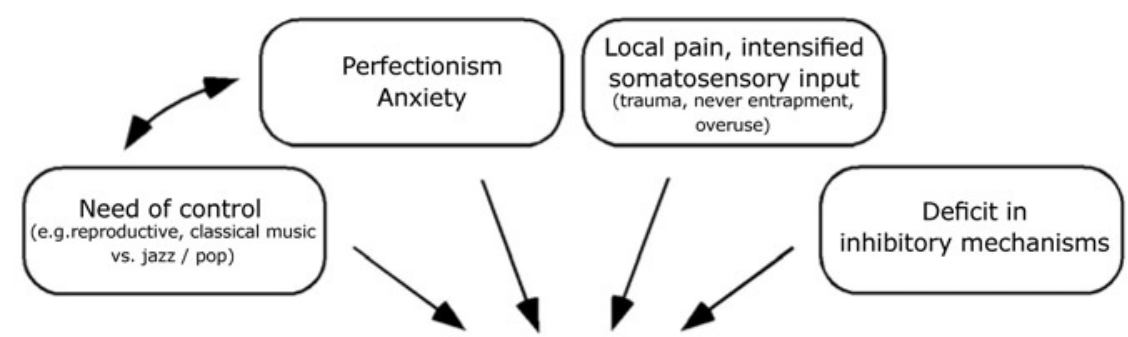

Intrinsic triggering factors

\section{Extrinsic triggering factors}

Predisposition - Susceptibility

a) male gender

b) hereditary component
Manifestation

of Musician's

Dystonia

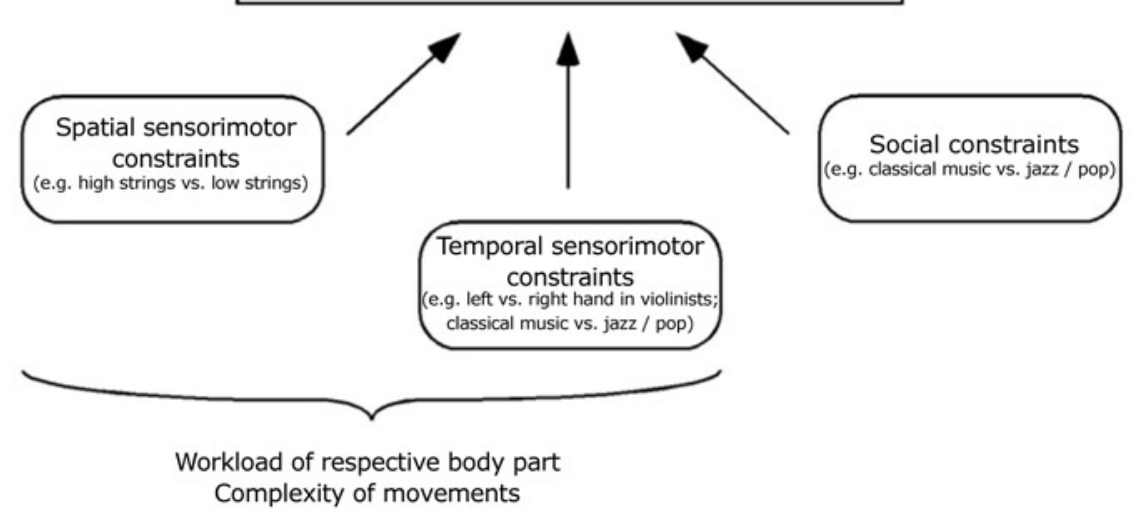

\section{Figure 4.}

The possible coaction between predisposition and intrinsic and extrinsic triggering factors in the manifestation of musician's dystonia. Further explanations are given in the text.

naire which was mailed to patients. Those patients who did not respond were contacted by phone, and a standardized interview was conducted using the questionnaire. Patients were asked to estimate 1 ) the cumulative treatment response on a four-step scale (free of symptoms - improved - no change - deteriorated); 2) the treatment response to individual therapies (improvement - no effect - deterioration). They were asked about 3) the nature and duration of therapies not applied by the authors (e.g. exercises on the instrument); 4) their musical activity and professional situation today. Outcome data of the aforementioned 144 patients could be obtained. Data were analyzed using $\chi^{2}$-tests for outcome measures, and multiple regression analyses for predictability of treatment responses. Percentage rates were calculated based on the total number of available answers.

\section{Longterm-outcome}

The mean age at the time of the completion of the questionnaire was 42 years (range 23-69 years). Outcome was revealed on average 8.4 years after onset of dystonic symptoms (range $1-30$ years) and 3.4 years after the initial visit (range 1-8 years). At the time of the survey, 77 patients (54\%) reported an alleviation of symptoms as compared to the time of their first visit, two of these $(1 \%)$ reported to be free of symptoms. Fifty patients $(35 \%)$ did not notice any changes and 16 patients $(11 \%)$ reported deterioration. Regardless of treatment, a smaller fraction of patients with embouchure dystonia showed an improvement ( 3 of 20 patients; $15 \%$ of these) compared to those with limb dystonia (62 of 123 patients; $\chi^{2}=8.7, d f=1, p<.01$ ). A change of profession was reported by 35 patients (29\%). A larger fraction of music students changed their profession (12 of 21 patients, 57\%) compared to those who were already in their professional careers at onset of symptoms (23 of 100 patients; $\chi^{2}=9.84$, $d f=1, p<.01$ ) (Jabusch et al., 2005).

\section{Trihexyphenidyl}

Trihexyphenidyl is an anticholinergic medication which is applied orally. Trihexyphenidyl was given to patients with all kinds of musician's cramps when no contraindication was present. Trihexyphenidyl was administered beginning with $1 \mathrm{mg} / \mathrm{d}$, and the dosage was slowly 
increased until therapeutic effects were observed. Adjustment of the dosage was made depending on beneficial effects and side effects. Patients with side effects and no improvement of symptoms were withdrawn from Trihexyphenidyl treatment.

Trihexyphenidyl was applied in 69 patients (48\% of all patients) with an average maximum dosage of $11 \mathrm{mg} / \mathrm{d}$ (range 1-30 mg/d). Within two months, the medication was interrupted in 20 patients due to side effects or unsatisfying treatment response. The other 49 patients (34\% of all) received Trihexyphenidyl in the average for 16 months (range 3-67 months). Most frequent side effects were dry mouth (29 patients; $42 \%$ of patients receiving Trihexyphenidyl), tiredness (15 patients; 22\%), dizziness (13 patients; 19\%), agitation (10 patients; 14\%), memory impairment (8 patients; 12\%), drowsiness (7 patients; 10\%), depression ( 7 patients; $10 \%$ ), loss of concentration (5 patients; 7\%), nausea (4 patients; 6\%), hyperkinesia (3 patients; 4\%), impaired visual accommodation (2 patients; $3 \%$ ), and tremor (2 patients; $3 \%$ ). The average minimum dosage at which side effects occurred was $9 \mathrm{mg} / \mathrm{d}$ (range $1-20 \mathrm{mg} / \mathrm{d}$ ). Due to side effects, 16 patients (23\%) were withdrawn from Trihexyphenidyl. Side effects were not related to age or gender. After Trihexyphenidyl, an improvement was reported by a total of 23 patients (33\%) and by 19 patients with limb dystonia who received Trihexyphenidyl for more than two months ( $42 \%$ of these; average duration 26 months). Patients' ratings of treatment results are displayed in Figure 5. None of the five patients with embouchure dystonia had an improvement after Trihexyphenidyl. Side effects occurred more often in patients who reported an improvement after Trihexyphenidyl (side effects present in 22 out of 23 patients) than in patients with no improvement (side effects present in 32 out of 44 patients: $\chi^{2}=6.66$, $d f=1, p<.01$ ). Trihexyphenidyl average maximum dosage was $11 \mathrm{mg} / \mathrm{d}$ (range 4-30 mg/d) in patients who had an improvement and $11 \mathrm{mg} / \mathrm{d}$ (range $1-20 \mathrm{mg} / \mathrm{d}$ ) in patients with no improvement. Multiple regression analysis revealed a model predicting $49 \%$ of the variance of patients' ratings of Trihexyphenidyl effects (R-square $=0.52 ;$ R-square adjusted $=0.49$ ). The following variables predicted a positive rating: limb localization of dystonia (vs. embouchure dystonia. Beta $=0.59 ; d f=1 ; F=37 ; p<.001)$, a high maximum dosage of Trihexyphenidyl (Beta $=0.28$; $d f=1 ; F=6.8 ; p=.01)$, a low number of concomitant treatments $($ Beta $=-0.35 ; d f=1 ; F=11$; $p<.01)$. The variables age, gender, localized vs. nonlocalized dystonia (localized dystonia affecting one or two fingers vs. non-localized dystonia), type of dystonia (flexion / extension / embouchure), task specificity at first visit, duration of dystonia were not sufficient predictors of the rating of Trihexyphenidyl effects.

The main predictors of an improvement after treatment with Trihexyphenidyl were the localization of dystonia in a limb, a high maximum dosage, and a low number of concomitant treatments. The low number of concomitant treatments may be a consequence of a successful treatment with Trihexyphenidyl in some patients which limits its value as a predictor variable. A high maximum dosage was a sufficient, but not a necessary condition for an improvement. One patient who tolerated a maximum dosage of $30 \mathrm{mg} / \mathrm{d}$ had an improvement, but in other patients an improvement was seen after low dosages. However, there was no patient with high maximum dosages in the group of non-responders to Trihexyphenidyl. Taken together, the applicability of Trihexyphenidyl was limited due to the frequent occurrence of side effects. These were more often reported by responders than by non-responders although both groups had the same average maximum dosage. All patients with embouchure dystonias were non-responders. Due to the easy handling, a treatment attempt with Trihexyphenidyl appears to be an option for musicians with limb dystonia when no contraindications are present (Jabusch et al., 2005).

\section{Botulinum Toxin}

After injection of a small dosage of Botulinum Toxin A (BT) into a muscle, the activity of the injected muscle is reduced by blocking the release of acetylcholine at the neuromuscular junction. This effect persists for a period of 4 to 6 months. BT injections were recommended only to those patients in which primary dystonic movements could be clearly distinguished from secondary compensatory movements. A lyophilized Botulinum Toxin A powder (Dysport ${ }^{\circledR}$, Ipsen Ltd., Berkshire, UK) was injected using an EMG-guided technique (Karp et al., 1994; Karp 2004; Schuele et al., 2005). Target muscles were identified by visual inspection of the dystonic movements while patients were playing their instruments. In patients with flexion or extension dystonia of individual fingers, injections in the forearm muscles were preferred. Additional injections in the hand muscles were performed in patients with dystonic flexion in the MCP joints and an extension component in the PIP and DIP joints.

BT injections were applied in 71 patients (49\%), three of whom had embouchure dystonia. Eighteen patients had only one injection due to unsatisfying 
No. of patients

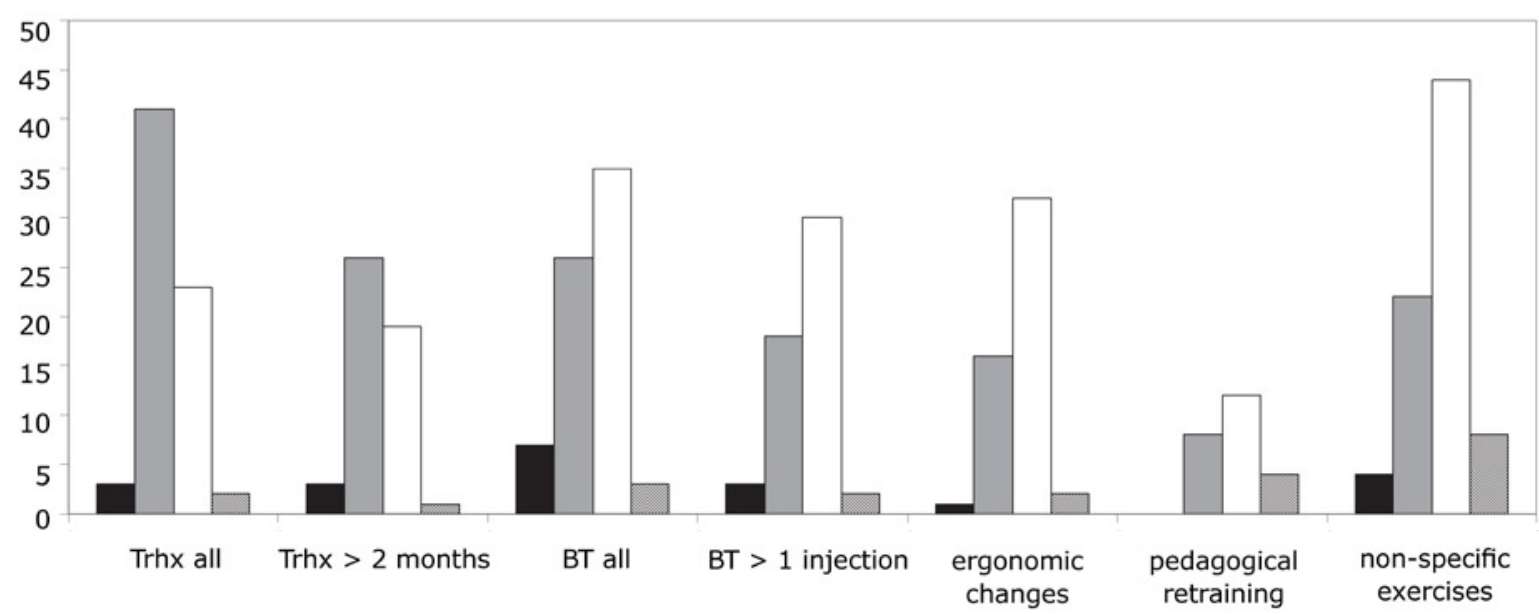

\section{Figure 5.}

Patients' rating of treatment results. Black bars: deterioration of dystonic symptoms. Grey bars: no change of dystonic symptoms. White bars: alleviation of dystonic symptoms. Hatched bars: no answer.

Trihexyphenidyl (Trhx): results are given separately for all patients who received Trhx vs. for those who received Trhx for more than two months.

Botulinum toxin (BT): results are given separately for all patients who received BT vs. for those who received more than one injection (from Jabusch et al., 2005).

treatment results. Five patients who reported an improvement were not satisfied enough to continue BT treatment. 53 patients (37\%) received more than one injection (average 5.7 injections per patients, range 2-25) with an average treatment duration of 16 months (range 1-58 months) and an average interval of 4.2 months (range 1-22 months) between treatment sessions. The average dosage per treatment session was 128 Units (range 9-428). The most injected muscles were the flexor digitorum superficialis and the flexor digitorum profundus (each: 50 patients; $70 \%$ of patients treated with BT), followed by the flexor carpi radialis (13 patients; $18 \%$ ), the flexor pollicis longus, the extensor digitorum and the extensor indicis ( 7 patients for each; $10 \%$ ), and the interosseus palmaris (5 patients; 7\%). The average dosage per muscle group was 112 Units (range 88-150) in the upper arm and shoulder muscles, 38 Units (range 5-85) in forearm extensors, 65 Units (range 10-175) in forearm flexors, 26 Units (range 5-84) in hand muscles. After BT, an improvement was reported by 35 patients (49\%) and by 30 patients who received more than one injection ( $57 \%$ of these; average duration 26 months). Of the latter group, 21 of 33 patients with injections given only in the forearm muscles reported an improvement $(64 \%)$, and 7 of 13 patients with injections in the forearm muscles and hand muscles (54\%) had an improvement: two out of two keyboard players, two of four woodwind players, and three of four guitarists with the right hand affected. No improvement was seen in the affected left hands of one guitarist and two string players after injections in the forearm muscles and hand muscles. None of two patients with injections in the upper arm / shoulder muscles experienced an improvement and none of three patients with embouchure dystonia experienced an improvement after BT. Multiple regression analysis did not reveal any of the following variables as predictors of outcome after BT: gender, age, duration of dystonia, localized vs. nonlocalized dystonia, type of dystonia, target muscles, mean BT dosage, task specificity at first visit, number of concomitant treatments. Positive rating of BT effects was correlated to the number of BT treatment sessions (Spearman $r=0.37, p<.01$ ) and the duration of BT treatment (Spearman $r=0.56, p<.01$ ).

According to these results, BT treatment was successful in those patients in which primary dystonic movements could be clearly distinguished from secondary compensatory movements. This was difficult when compensatory movements were more pronounced than primary dystonic movements. EMG-guidance was always applied and seems mandatory since a previous study has demonstrated that in the absence of EMG-guidance, only $37 \%$ of the needle placements reached the desired muscles (Molloy et al. 2002). In our patient group, the best outcome was reported after injections in forearm muscles. Additional injections in hand muscles were useful in patients with a dystonic flexion in the MCP joints and an extension component in the PIP and DIP joints. BT injections in hand muscles 
appear to be recommendable only in instrumentalists with little lateral finger motion such as in woodwind players and guitarists (right hand). Keyboard players may benefit from this option when they avoid repertoire requiring a wide hand span and extreme lateral finger motion. BT injections in hand muscles were not successful in musicians who needed to perform lateral finger movements such as in the left hands of one guitarist and of two string players. Musicians with embouchure dystonia and such with dystonia affecting the upper arm and shoulder muscles did not benefit from BT treatment (Jabusch et al. 2005).

\section{Ergonomic changes}

Ergonomic changes were recommended whenever applicable. The aim of ergonomic changes was either a blocking of dystonic movements, for example by attaching splints to the affected fingers. Alternatively, circumvention of dystonic movements was achieved by modifications of the instrument, e.g. the repositioning of individual keys in woodwind instruments or the replacement of ring keys by plateau keys (Altenmüller, 2003). Support systems (belts, stands, tripods) were recommended when the dystonic hand had to carry the instrument while playing such as in oboe-, clarinet- , bassoon players. Ergonomic changes were applied in 51 patients ( $35 \%$ of all), 32 patients ( $63 \%$ of these) experienced improvement and used the ergonomic changes for an average of 35 months (range 3-125 months). Two patients with embouchure dystonia used an ergonomic aid and reported no improvement. As a conclusion, ergonomic changes should be considered in all patients with limb dystonia. They are not helpful in embouchure dystonia (Jabusch et al., 2005).

\section{Pedagogical retraining and unspecific exercises}

Pedagogical retraining was applied in patients with all forms of musician's dystonia. They comprised a variety of behavioral approaches which were taking place under the supervision of instructors and included elements based on the following principles reported previously (Byl et al., 2000; Boullet, 2003; Candia et al. 1999; Candia et al., 2002): 1) movements of affected body parts were limited to a level of tempo and force at which the dystonic movement would not occur; 2) compensatory movements (e.g. of adjacent fingers) were avoided, partially under the application of splints; 3 ) instant visual feedback with mirrors or monitors helped patients to recognize dystonic and non-dystonic movements; 4) body awareness techniques (e.g. Feldenkrais ${ }^{\circledR}$ ) were applied to increase the patient's perception of non-dystonic movements.

Pedagogical retraining was applied in 24 patients ( $17 \%$ of all), 12 patients ( $50 \%$ of these) experienced improvement. Patients reporting an improvement had undergone this treatment for an average of 28 months (range 3-72 months). Two patients with embouchure dystonia took part in pedagogical retraining and reported no improvement.

Besides of the reported therapies recommended by the authors, patients additionally or alternatively performed unspecific technical exercises on their instruments which are usually practiced by instrumentalists to improve their technique. 78 patients (54\% of all) were practicing such unspecific technical exercises, 44 patients ( $56 \%$ of these) experienced improvement. Six out of 11 patients with embouchure dystonia reported an improvement after practicing such exercises (Jabusch et al., 2005).

These results provide evidence for the benefit of a behavioral approach, either by pedagogical retraining or by exercises on the instrument. Pedagogical retraining was applied in patients with all forms of musician's dystonia, however, only patients with limb dystonia improved. More than half of the patients who were practicing unspecific exercises reported improvement, among them were six out of 11 patients with embouchure dystonia who were engaged in such exercises. It is noteworthy that the results of these exercises were rated better than that of medical therapies. These positive results may be biased by a particular attitude among musicians and the perceived self-control through exercises. On the other hand, positive results of retraining and of exercises underline the benefit of an active involvement of patients in the treatment process. At present, the existing behavioral approaches are heterogeneous (Byl et al., 2000; Boullet, 2002; Candia et al., 2002). Instrument-specific retraining programs ought to be elaborated which include the aforementioned principles. All approaches of retraining therapy require time and patience on the part of the affected musician, which seem to be limiting factors of these therapies. Behavioral aspects, however, should be included in all treatment approaches, at least as an adjunct.

\section{Findings in the light of the literature}

With regard to the overall outcome, which was recorded on average 8.4 years after the onset of symp- 
toms, $71 \%$ of patients managed to stay in their musical profession. In other studies, focal dystonia had led to the end of the musical career in more than half of the patients (Schuele \& Lederman 2003, 2004). Outcome measures were conducted 13.8 (string instrumentalists) (Schuele \& Lederman 2004) and 8.5 years (woodwind players) (Schuele \& Lederman 2004) after the onset of symptoms in these reports. The shorter duration of dystonia might be an explanation for the better outcome of our patient group compared to the report on the string players. In the study on woodwind players, the rate of patients with embouchure dystonia was $25 \%$ which differs from our group (13.9\%). A generally worse outcome in embouchure dystonia might explain the different outcome after similar durations of dystonia in both studies. In another report, 17 out of 33 musicians with focal dystonia had stopped playing at the end of the follow-up period (Brandfonbrener \& Robson, 2002). Duration of dystonia and total followup time were, however, not mentioned which did not allow a direct comparison. This was also the case in a report of 145 musicians with an average rehabilitation time of 24 months, after which 35 musicians returned to playing in public (Tubiana \& Chamagne, 2000).

\section{Psychotherapy}

Since psychological conditions such as perfectionism and anxiety were found to be related to musician's dystonia, psychotherapy has been recommended to be included in the treatment (Chamagne, 2003). Some patients suffer dramatically from the loss of their abilities on the instrument and from the whole situation. Psychotherapy may be helpful for such patients to find coping strategies and to handle the situation. In order to improve the preconditions for an effective application of other therapies, the authors occasionally recommended psychotherapy to patients with these secondary psychoreactive phenomenons. The direct effects of psychotherapy on the motor coordination of patients with musician's dystonia, especially in those with extreme perfectionism or with anxious traits, have not yet been investigated.

\section{Instrumental students}

The subgroup of instrumental students deserve some additional consideration. Focal dystonia is regarded as incurable at present. Although there are some patients with encouraging follow-up results, therapy remains difficult and requires years and sometimes decades of patience, effort and stress. Most patients who are at the zenith of their career have no choice and have to undergo treatment in order to improve their situation. Instrumental students, in contrary, are young and flexible enough to change their profession. In our group, 12 out of 21 affected students changed their profession during the follow-up period. According to our impression, therapy results do not justify keeping students busy with treatment attempts over years in the most flexible and productive period of their lives. We conclude that strategies for students with musician's dystonia should include early support and encouragement to change to another profession.

\section{Limitations}

Besides the reported inclusion / exclusion criteria, the choice of treatment was strongly influenced by preferences of patients. Time investment appeared to influence the choice of treatment. Older patients who desired a quick improvement preferred BT injections. In contrast, several patients found the injection therapy 'unnatural' and preferred a retraining therapy. A major limitation of the study was the lack of objective assessment tools for musician's dystonia which were not available at the beginning of the follow-up. Recently, reliable and precise objective methods have been developed for quantification of musician's dystonia (Jabusch \& Altenmüller, 2004b; Jabusch et al., 2004b). In future studies, such methods will have to be applied for reliable monitoring of treatment effects. Although we were limited in our assessment to a retrospective and subjective rating, several considerations support the validity of our result. Consistent negative results reported by patients with embouchure dystonia after treatment with BT, Trihexyphenidyl and retraining seem to indicate a low placebo-sensitivity of musician's dystonia. Most previous studies observed that a significant number of musicians discontinued treatment within a year if they felt the response did not meet their needs. The adherence to treatment seen in our study, with an average treatment duration in responders between two and three years (and up to ten years), seems unlikely that the result did not improve their instrumental performance significantly.

\section{CONCLUSION}

Focal dystonia in musicians is still the main challenge in Musicians' Medicine. With the available therapies, the majority of patients manage to stay in their profession, many of them, however, with substantial compromises. Therapy of patients with embouchure 
dystonia remains problematic. The available medical approaches are ineffective, and other options have yet to be developed. There is a pressing need for novel therapies for musician's dystonia. As a new method, immobilization of affected limbs for four to five weeks with a splint was reported (Priori et al., 2001). This treatment resulted in a reduced severity of symptoms and improved performance in seven patients with musician's cramp and one patient with writer's cramp four weeks after removal of the splint, after 24 weeks improvement was still observed in seven patients. As another experimental approach, oral medication with $5 \mathrm{mg} \Delta 9$-Tetrahydrocannabinol (the major psychoactive ingredient of Cannabis sativa $L$.) in a pianist resulted in significant improvement of the pianistic performance for a period of 5 hours (Jabusch et al., 2004c). These treatment approaches have to be investigated in larger numbers of patients in order to confirm beneficial effects. Behavioral therapies and interdisciplinary strategies combining pharmacological and pedagogical methods are promising, but the different approaches need to be evaluated. Since phenomenological and epidemiological data and results from electrophysiological studies and from follow-up studies imply a behavioral component in the development as well as in the treatment of musician's dystonia, future research is required to identify 'beneficial behavior' on the instrument. This may possibly also be of help for finding strategies with the particular aim of preventing musician's dystonia.

\section{Acknowledgement}

A modified version of this review appeared in: Altenmüller E., Kesselring J., Wiesendanger M., eds. Music, Motor Control and the Brain. Oxford University Press, 2006.

\section{References}

Altenmüller, E. (2003). Focal dystonia: advances in brain imaging and understanding of fine motor control in musicians. Hand Clin., 19, 523-538

Bara-Jimenez, W., Catalan, M. J., Hallett, M., Gerloff, C. (1998). Abnormal somatosensory homunculus in dystonia of the hand. Ann. Neurol., 44, 828-831 [www

Boullet, L. (2003). Is there a cure for focal dystonia? Experiences with a new retraining therapy for pianists. Proceedings of the German Society for Music Physiology and Performing Arts Medicine, 9, 17

Brandfonbrener, A. G. (1995). Musicians with focal dystonia: A report of 58 cases seen during a tenyear period at a performing arts medicine clinic. Med. Probl. Perform. Art., 10, 121-127
Brandfonbrener, A. G., Robson, C. (2002). A review of 111 musicians with focal dystonia seen at a performing artist's clinic 1985-2002. Mov. Disord., 17, 1135 Byl, N. N., Merzenich, M. M., Jenkins, W. M. (1996). A primate genesis model of focal dystonia and repetitive strain injury: I. Learning-induced dedifferentiation of the representation of the hand in the primary somatosensory cortex in adult monkeys. Neurology, 47, 508-520 www

Byl, N. N., McKenzie, A. (2000). Treatment effectiveness for patients with a history of repetitive hand use and focal hand dystonia: a planned, prospective follow-up study. J. Hand Ther., 13, 289-301

Candia, V., Elbert, T., Altenmüller, E., Rau H., Schäfer, T., Taub, E. (1999). A constraint-induced movement therapy for focal hand dystonia in musicians. Lancet, 353, 42 WwW

Candia, V., Schafer, T., Taub, E., Rau, H., Altenmüller, E., Rockstroh B., Elbert T. (2002). Sensory motor retuning: a behavioral treatment for focal hand dystonia of pianists and guitarists. Arch. Phys. Med. Rehabil., 83, 1342-1348. wWw

Candia, V., Wienbruch, C., Elbert, T., Rockstroh, B., Ray W. (2003). Effective behavioral treatment of focal hand dystonia in musicians alters somatosensory cortical organization. Proc. Natl. Acad. Sci. USA, 100, 7942-7946. $|w w|$

Chamagne, P. (2003). Functional dystonia in musicians: rehabilitation. Hand Clin., 19, 309-316. WwW

Charness, M. E., Ross, M. H., Shefner, J. M. (1996). Ulnar neuropathy and dystonic flexion of the fourth and fifth digits: clinical correlation in musicians. Muscle Nerve, 19, 431-437. |www

Cole, R. A., Cohen, L. G., Hallett, M. (1991). Treatment of musician's cramp with Botulinum Toxin. Med. Probl. Perform. Art., 6, 137-143

Deuschl, G., Toro, C., Matsumoto, J., Hallett, M. (1995). Movement-related cortical potentials in writer's cramp. Ann. Neurol., 38, 862-868 [wWw

Elbert T., Candia, V., Altenmüller, E., et al. (1998). Alteration of digital representations in somatosensory cortex in focal hand dystonia. Neuroreport, 9, 3571-3575 [wW

Fletcher, N. H., Rossing, T. D. (1991). The Physics of Musical Instruments. New York, Springer.

Flor, H., Braun, C., Elbert, T., Birbaumer, N. (1997). Extensive reorganization of primary somatosensory cortex in chronic back pain patients. Neurosci. Lett., 224, 5-8 [Ww|

Frucht, S. J., Fahn, S., Greene, P. E. (2001). The natural history of embouchure Dystonia. Mov. Disord., $16,899-906$ WwW 
Gembris H., Langner D. (2005). Von der Musikhochschule zum Arbeitsmarkt (From Music Academy to the Job Market). Kraemer RD (ed.) Augsburg, WissnerVerlag. pp 71-72

Hallett M. (1998). The neurophysiology of dystonia. [Review]. Arch. Neurol., 55, 601-603 www

Hua S. E., Garonzik I. M., Lee J. I., Lenz F. A. (2000). Microelectrode studies of normal organization and plasticity of human somatosensory thalamus. J. Clin. Neurophysiol., 17, 559-574

Hummel F., Andres F., Altenmüller E., Dichgans J., Gerloff C. (2002). Inhibitory control of acquired motor programmes in the human brain. Brain 125, 404420. $\mid \underline{W W}$

Jabusch H. C., Altenmüller E. (2004a). Anxiety as an aggravating factor during onset of focal dystonia in musicians. Med. Probl. Perform. Art., 19, 75-81

Jabusch H. C., Altenmüller E. (2004b). Three-dimensional movement analysis as a promising tool for treatment evaluation of musicians' dystonia. Adv. Neurol., 94, 239-45

Jabusch H. C., Müller S. V., Altenmüller E. (2004a). Anxiety in musicians with focal dystonia and those with chronic pain. Mov. Disord., 19, 1169-1175 [www

Jabusch H. C., Vauth H., Altenmüller E. (2004b). Quantification of focal dystonia in pianists using Scale Analysis. Mov. Disord., 19, 171-180 Www

Jabusch H. C. , Schneider U. , Altenmüller E. (2004c). $\Delta^{9}$-Tetrahydrocannabinol (THC) improves motor control in a patient with musician's dystonia. Mov. Disord., 19, 990-991

Jabusch H. C., Zschucke D., Schmidt A., Schuele S., Altenmüller E. (2005). Focal dystonia in musicians: treatment strategies and long-term outcome in 144 patients. Mov. Disord., 20, 1623-1626 [www

Jankovic J., Shale H. (1989). Dystonia in musicians. Semin. Neurol., 9, 131-135 www

Karp B. I., Cole R. A., Cohen L. G., Grill S., Lou J. S., Hallett M. (1994). Long-term botulinum toxin treatment of focal hand dystonia. Neurology, 44, 70-76. WWW

Karp B. I. (2004). Botulinum toxin treatment of occupational and focal hand dystonia. Mov. Disord., 19 Suppl, 8, 116-119. www

Lederman R. J. (1991). Focal dystonia in instrumentalists: clinical features. Med. Probl. Perform. Art., 6, 132-136

Lenz F. A., Byl N. N. (1999). Reorganization in the cutaneous core of the human thalamic principal somatic sensory nucleus (Ventral caudal) in patients with dystonia. J. Neurophysiol., 82, 3204-3212 www
Lim V. K., Altenmüller E. (2003). Musicians' cramp: Instrumental and gender differences. Med. Probl. Perform. Art., 18, 21-26

Molloy F. M., Shill H. A., Kaelin-Lang A., Karp B. I. (2002). Accuracy of muscle localization without EMG: Implications for treatment of limb dystonia. Neurology, 58, 805-807 [ww]

Naumann M., Pirker W., Reiners K., Lange K. W., Becker G., Brucke T. (1998). Imaging the pre- and postsynaptic side of striatal dopaminergic synapses in idiopathic cervical dystonia: a SPECT study using [123I] epidepride and [123I] beta-CIT. Mov. Disord.,

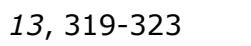

Preibisch C., Berg D., Hofmann E., Solymosi L., Naumann M. (2001). Cerebral activation patterns in patients with writer's cramp: a functional magnetic resonance imaging study. J. Neurol., 248, 10-17 WWW Priori A., Pesenti A., Cappellari A., Scarlato G., Barbieri S. (2001). Limb immobilization for the treatment of focal occupational dystonia. Neurology, 57, 405-409 WWW

Pujol J., Roset-Llobet J., Rosines-Cubells D., et al. (2000). Brain cortical activation during guitar-induced hand dystonia studied by functional MRI. Neuroimage, 12, 257-267 Www

Rosenkranz K., Williamon A., Butler K., Cordivari C., Lees A. J., Rothwell J. C. (2005). Pathophysiological differences between musician's dystonia and writer's cramp. Brain doi:10.1093/brain/awh402 [WwW

Schmidt A., Jabusch H. C., Altenmüller E., Hagenah J., Brüggemann N., Hedrich K., Saunders-Pullman R., Bressman S. B., Kramer P. L., Klein C. (2006). Dominantly transmitted focal dystonia in families of patients with musician's cramp. Neurology, 67, 691693 WWW

Schuele S., Lederman R. J. (2003). Focal dystonia in woodwind instrumentalists: long-term outcome. Med. Probl. Perform. Art., 18, 15-20

Schuele S., Lederman R. J. (2004). Long-term outcome of focal dystonia in string instrumentalists. Mov. Disord., 19, 43-8 www

Schuele S., Jabusch H. C., Lederman R. J., Altenmüller E. (2005). Botulinum toxin injections in the treatment of musician's dystonia. Neurology, 64, 341-343. Www

Toro C., Deuschl G., Hallett M. (2000). Movement-related electroencephalographic desynchronization in patients with hand cramps: evidence for motor cortical involvement in focal dystonia. Ann. Neurol., 47, 456-461. |www

Tubiana R., Chamagne P. (2000). Prolonged rehabilitation treatment of musician's focal dystonia. In: Tubiana R., Amadio P. C. (eds.) Medical problems of 
the instrumentalist musician. London, Martin Dunitz Ltd. pp 369-378

Tinazzi M., Fiaschi A., Rosso T., Faccioli F., Grosslercher J., Aglioti S. M. (2000). Neuroplastic changes related to pain occur at multiple levels of the human somatosensory system: A somatosensory-evoked potentials study in patients with cervical radicular pain. $J$. Neurosci., 20, 9277-9283. Www 